\section{REFERENCES}

Ball, S., Collins, F. D., Dalvi, P. D. \& Morton, R. A. (1949). Biochem. J. 45, 304.

Ball, S., Goodwin, T. W.\& Morton, R.A. (1948). Biochem.J. 42, 516.

Ball, S. \& Morton, R. A. (1949). Biochem. J. 45, 298.

Barany, H. C., Braude, E. A. \& Pianka, M. (1949). J. chem. Soc. p. 1898.

Brüggeman, J., Krauss, W. \& Tiews, J. (1952). Chem. Ber. 85, 315.

Cama, H. R., Collins, F. D. \& Morton, R. A. (1951). Biochem. J. 50, 48.

Collins, F. D. (1953). Nature, Lond., 171, 469.

Collins, F. D., Green, J. N. \& Morton, R. A. (1954). Bioch. J. 56, 493.

Collins, F. D., Love, R. M. \& Morton, R. A. (1952). Biochem. J. 51, 292.

Collins, F. D. \& Morton, R. A. (1950). Biochem. J. 47, 10.
Decker, H. \& Becker, P. (1913). Liebigs Ann. 295, 362.

Dewar, M. J. S. (1949). The Electronic Theory of Organic Chemistry, chap. 15. Oxford: Clarendon Press.

Farrar, K. R., Hamlet, J. C., Henbest, H. B. \& Jones, E. R. H. (1952). J. chem. Soc. p. 2657.

Hausser, K. W., Kuhn, R., Smakula, A. \& Hoffer, M. (1935). Z. phys. Chem. B 29, 371.

Hubbard, R. (1954). J. gen. Physiol. 37, 381.

Hubbard, R., Gregerman, R. I. \& Wald, G. (1953). J. gen. Physiol. 36, 415.

Hubbard, R. \& Wald, G. (1952). J. gen. Physiol. 36, 269.

Lythgoe, R. J. (1937). J. Physiol. 89, 331.

Meunier, P. (1942). C.R. Acad. Sci., Paris, 215, 470.

Morton, R. A. \& Goodwin, T. W. (1944). Nature, Lond., $153,405$.

Morton, R. A. \& Pitt, G. A. J. (1955). Biochem. J. 59, 128.

Wald, G. (1935). J. gen. Physiol. 19, 351.

Wald, G. (1948). J. gen. Physiol. 31, 489.

Wald, G. (1953). Annu. Rev. Biochem. 22, 497.

Wald, G. \& Brown, P. K. (1953). J. gen. Physiol. 37, 189.

\title{
Studies on Rhodopsin
}

\section{9. pH AND THE HYDROLYSIS OF INDICATOR YELLOW}

\author{
By R. A. MORTON ANd G. A. J. PITT \\ Department of Biochemistry, The University of Liverpool
}

(Received 16 June 1954)

Lythgoe (1937) showed that on exposure to light, rhodopsin (visual purple) solutions break down to give the pigment 'indicator yellow', which has $\lambda_{\max .}$ about $365 \mathrm{~m} \mu$. in alkali ('alkaline' indicator yellow) and about $440 \mathrm{~m} \mu$. in acid ('acid' indicator yellow) (Collins \& Morton, 1950).

The $\mathrm{pH}$ affects not only the spectrum but also the stability of indicator yellow. Lythgoe (1937) found his preparations to be stable above $\mathrm{pH} 7$ in the alkaline form and fairly stable in the acid form at pH 3.3. Between $\mathrm{pH} 4 \cdot 0$ and 6.1, however, the acid form decomposed to give what Bliss (1948) later identified as retinene (vitamin $\mathbf{A}$ aldehyde). To explain this behaviour Lythgoe postulated two forms of acid indicator yellow-one stable (existing at $\mathrm{pH} 3 \cdot 3$ ), the other unstable (existing between pH 4.0 and 6.1). Wald (1938), Bliss (1948) and Collins \& Morton (1950) have reported acid indicator yellow as unstable, but they all studied indicator yellow solutions near $\mathrm{pH} 4 \cdot 0$, i.e. they were dealing with the unstable form. Lythgoe's stable acid indicator yellow has largely been overlooked.

Substances behaving spectroscopically as indicator yellow, and known as indicator yellow analogues, have been made by the interaction of retinene and amines (Ball, Collins, Dalvi \& Morton, 1949). These are Schiff's bases-retinylideneamines-which in acid solution form retinylidene- ammonium salts (using the nomenclature of Pitt, Collins, Morton \& Stok, 1955). It has been observed in this laboratory (F.D.Collins, unpublished) that, in fairly strongly acid solutions, these indicator yellow analogues are stable, in the sense that they do not hydrolyse to retinene. The availability of retinylidenemethylamine (a crystalline indicator yellow analogue; Pitt et al. 1955) allows its stability to be studied in aqueous solution without interference caused by other substances inevitably present in solutions of natural indicator yellow obtained from eyes.

Such a study is reported in this paper and, based on the knowledge thus obtained, further investigations have been carried out of the relationship between indicator yellow and rhodopsin.

\section{EXPERIMENTAL}

Absorption spectra were determined by means of a photoelectric spectrophotometer.

Retinylidenemethylamine. This was prepared by the method of Pitt et al. (1955). The crystalline material was used.

Aqueous solutions of retinylidenemethylamine. These were made using Tween 80 (polyoxyethylene derivative of sorbitan mono-oleate, Honeywill and Stein, Ltd., St James' Square, London, S.W. 1) by the technique of Bliss (1951) and were of concentrations suitable for reading in the spectrophotometer (approx. $\mathrm{M} \times 10^{-5}$ ). 
In this study, Tween 80 is more convenient than digitonin, which is accepted as the best aid to the extraction of rhodopsin and thence of indicator yellow. Digitonin slowly precipitates from aqueous solutions (particularly when acid), thereby causing turbidity and interference with spectrophotometric measurements. The substances with which this investigation is concerned have their spectra in Tween 80 solutions moved to shorter wavelengths as compared with those found in digitonin solutions. The differences are summarized in Table 1. (This shift is responsible for one of the apparent differences between indicator yellow and retinylidenemethylamine reported by Collins (1953) because he studied indicator yellow in digitonin solution, and retinylidenemethylamine in Tween 80.) Retinylidenemethylamine solutions of different $\mathrm{pH}$ values were made using: $\mathrm{pH}$ values 1 and $2, \mathrm{HCl} ; 3$, acetic acid; 3.7 and 4.8 acetate buffer; $5 \cdot 7$, phthalate buffer; $6 \cdot 3,6 \cdot 6$ and $7 \cdot 7$, phosphate buffer; $8 \cdot 6$, borate buffer; $13, \mathrm{KOH}$. The $\mathrm{pH}$ of the resulting solution was checked at the end of the experiment, using a Lovibond comparator, except for those of $\mathrm{pH}$ values 1,2 and 13, which were made using standardized acid and alkali.

Rhodopsin. Solutions were prepared from ox retinas by a slight modification of the method of Collins, Love \& Morton (1952). After the alum treatment, the rods were washed with $0.67 \mathrm{M}$ phosphate buffer of $\mathrm{pH} \mathrm{6.3} \mathrm{(Clark,}$ 1928), mixed with anhydrous $\mathrm{Na}_{2} \mathrm{SO}_{4}$, extracted twice with light petroleum (b.p. 40-60') and washed with water until free from sulphate. The rhodopsin was then extracted with a $1 \%(w / v)$ solution of digitonin in $0.67 \mathrm{M}$ phosphate buffer (pH 6.3).

Acid destruction of rhodopsin. The absorption spectrum of the rhodopsin solution $(2 \mathrm{ml}$.) was recorded. $\mathrm{HCl}(0.03 \mathrm{ml}$., $10 \mathrm{~N})$ was added in the dark, and the absorption spectrum examined rapidly. KOH $(0.05 \mathrm{ml} ., 30 \%, \mathrm{w} / \mathrm{v})$ and $0.5 \mathrm{ml}$. of approx. 1.5 M methylamine containing excess $\mathrm{KOH}$ were added. After $5 \mathrm{~min}$., the mixture was reacidified with $0.1 \mathrm{ml} .10 \mathrm{~N}-\mathrm{HCl}$ and the absorption curve redetermined.

\section{RESULTS}

\section{$\mathrm{pH}$ and hydrolysis of retinylidenemethylamine}

Aqueous solutions of retinylidenemethylamine were made at different $\mathrm{pH}$ values. The absorption spectra were recorded immediately after mixing and observations continued so that any change could be followed. In most cases $\lambda_{\max }$ moved to 380-385 $\mathrm{m} \mu$, showing that retinylidenemethyl-

Table 1. Effect of Tween 80 and digitonin on $\lambda_{\max }$ of aqueous dispersions

\begin{tabular}{|c|c|c|}
\hline Compound & In digitonin & In Tween 80 \\
\hline Retinene* & 389 & 383 \\
\hline $\begin{array}{l}\text { Retinylidenemethylamine } \\
\text { (in alkali) }\end{array}$ & 365 & 362 \\
\hline $\begin{array}{l}\text { Retinylidenemethylamine } \\
\text { (in acid) }\end{array}$ & 438 & 430 \\
\hline
\end{tabular}

* $\lambda_{\max }$ of retinene in aqueous solutions is not changed in acid or alkali. amine was being hydrolysed to retinene. The time taken for $\lambda_{\max }$. to change to $380-385 \mathrm{~m} \mu$. was noted. Results are shown in Table 2.

At pH 1.0 no hydrolysis of retinylidenemethylammonium chloride occurred. The absorption curve observed immediately on mixing, and that after standing at room temperature for $18 \mathrm{hr}$. are shown in Fig. 1. After $18 \mathrm{hr}$. $\lambda_{\max }$ was still at $430 \mathrm{~m} \mu$., but there was a general fall in extinction

\section{Table 2. Hydrolysis of retinylidenemethylamine in aqueous solution}

Tween 80 used as dispersing agent.

$\begin{array}{ccc}\text { pH } & \begin{array}{c}\lambda_{\max }(\mathrm{m} \mu .) \\ \text { immediately } \\ \text { after mixing }\end{array} & \begin{array}{c}\lambda_{\text {max }} \text { moved to } \\ \mathbf{3 8 0}-\mathbf{3 8 5} \mathrm{m} \mu . \\ \text { after }\end{array} \\ 1.0 & 430 & \text { No change } \\ 2 \cdot 0 & 430 & 30 \mathrm{hr} . \\ 3 \cdot 0 & 430 & \text { Approx. } 10 \mathrm{hr} . \\ 3 \cdot 7 & 428 & 90 \mathrm{~min} . \\ 4 \cdot 8 & 415 & 35 \mathrm{~min} . \\ 5 \cdot 7 & 397 & 10 \mathrm{~min} . \\ 6 \cdot 6 & 367 & 28 \mathrm{~min} . \\ 7 \cdot 7 & 363 & 75 \mathrm{~min} . \\ 8 \cdot 6 & 363 & 100 \mathrm{~min} . \\ 13 & 363 & 110 \mathrm{~min} .\end{array}$

In the presence of $0.2 \mathrm{M}$ methylamine

$\begin{array}{ccc}3 \cdot 7 & 424 & 90 \mathrm{~min} . \\ 6 \cdot 3 & 366 & 60 \mathrm{~min} . \\ 8 \cdot 6 & 362 & \text { No change }\end{array}$

In the presence of $4 \%(w / v)$ aqueous formaldehyde $\mathbf{8 . 6} \quad 370 \quad 9$ min.

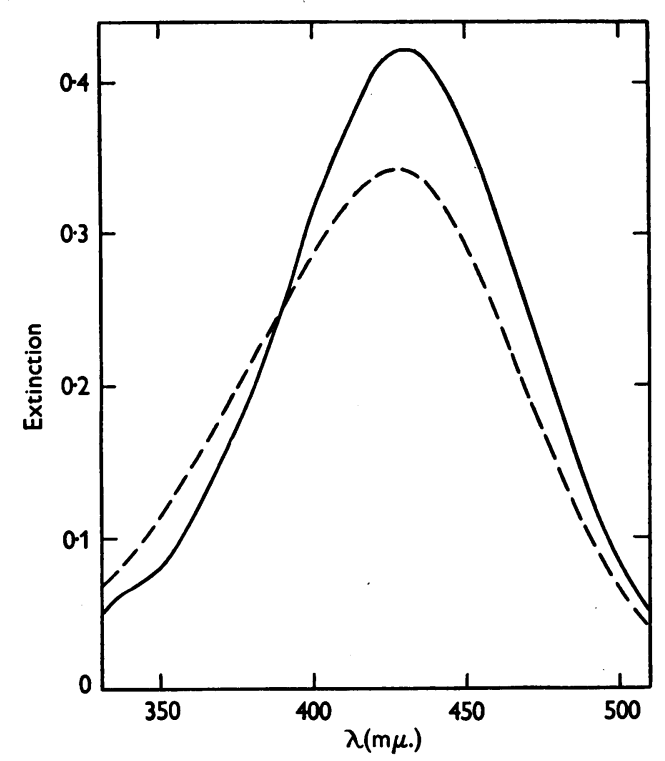

Fig. 1. Absorption spectrum of retinylidenemethylamine in $0.1 \mathrm{~N}-\mathrm{HCl}$ : on mixing, _persing agent-Tween 80.)

Bioch. 1955, 59 
at wavelengths above $390 \mathrm{~m} \mu$. and a rise at shorter wavelengths. This is presumably due to nonhydrolytic destruction of retinylidenemethylammonium chloride, such as commonly occurs with derivatives of vitamin $A$ in polar solvents at room temperature, particularly when exposed to air. Also acid tends to isomerize carotenoids (Zechmeister, 1944) and may well isomerize retinylidenemethylammonium chloride in these conditions, causing a drop in extinction. There is, however, no indication of any measurable hydrolysis to retinene, which would be marked by a peak or rise in extinction at $383 \mathrm{~m} \mu$.

At $\mathrm{pH}$ values of $2 \cdot 0$ and $3 \cdot 7$, the retinylidenemethylammonium salt initially formed hydrolyses to retinene-being less stable at the higher $\mathrm{pH}$. Fig. 2 shows the hydrolysis at pH 3.7. There is an isosbestic point at $400 \mathrm{~m} \mu$., indicating that the effective absorption is due to only two substances.

At $\mathrm{pH}$ values 4.8 and $5 \cdot 7$, the initial $\lambda_{\max }$ lies between the maxima of retinene and the retinylidenemethylammonium salt, and very rapidly changes over to give the curve characteristic of retinene. The same changes are seen as at $\mathrm{pH} \mathrm{3.7,}$ but are proceeding faster so that the retinylidenemethylammonium salt has partially hydrolysed before it can be examined in the spectrophotometer.

The solution at $\mathrm{pH}$ 6.6 shows a maximum between those of retinylidenemethylamine (the uncharged Schiff's base) and retinene. At $\mathrm{pH}$ values above this, retinylidenemethylamine is more stable and hydrolyses more slowly to retinene. A figure showing the hydrolysis of retinylidenemethylamine has been given by Collins (1953).

When this behaviour of retinylidenemethylamine is compared with that of indicator yellow formed from rhodopsin solution (Lythgoe, 1937), it is seen that the stability of the acid indicator yellow

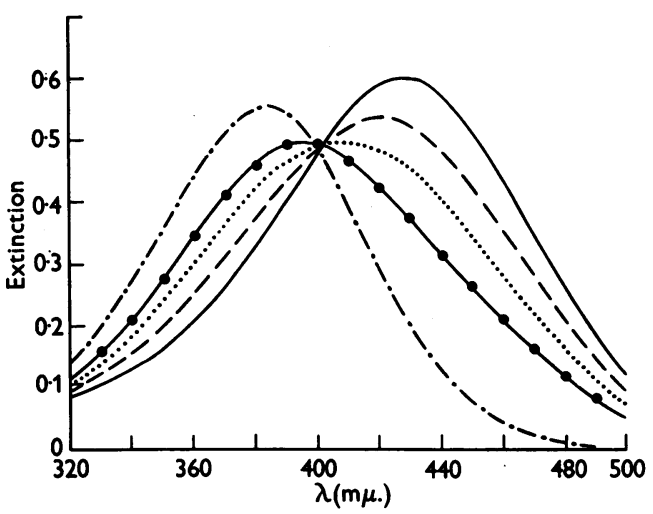

Fig. 2. Absorption spectrum of retinylidenemethylamine in aqueous solution at $\mathrm{pH} \mathrm{3.7:} \mathrm{on} \mathrm{mixing,} \mathrm{-} \mathrm{—} \mathrm{;} \mathrm{after}$ 12 min., - - ; after 20 min., ... ; after 35 min., $\bullet-\bullet$; after 75 min., ---•-. (Dispersing agent-Tween 80.) reported by Lythgoe is paralleled by the resistance of retinylidenemethylammonium chloride to hydrolysis at pH 1. Similarly, the hydrolysis of acid indicator yellow in a less acid $\mathrm{pH}$ range agrees with the behaviour of the analogue. However, hydrolysis of retinylidenemethylamine still occurs in alkaline solution (previously reported by Collins, 1953), whereas in solutions prepared from the eye, alkaline indicator yellow is stable.

This difference in behaviour can be explained by considering the hydrolysis of retinylideneamines to retinene and the free amine:

$$
\mathrm{C}_{19} \mathrm{H}_{27} \mathrm{CH}=\mathrm{N} \cdot \mathrm{R}+\mathrm{H}_{2} \mathrm{O} \rightleftharpoons \mathrm{C}_{19} \mathrm{H}_{27} \mathrm{CHO}+\mathrm{R} . \mathrm{NH}_{2} \text {. }
$$

In solutions of retinylidenemethylamine $\left(\mathrm{R}=\mathrm{CH}_{3}\right)$ and retinylideneamines in general, equilibrium (1) favours production of retinene at the concentrations used for spectrophotometry. By greatly increasing the concentration of methylamine it is possible to push the equilibrium in favour of Schiff's base formation, i.e. the method used by Ball et al. (1949) in their original preparation of retinylidenemethylamine. Ball et al. (1949) used an aqueous solution containing ethanol, but this was found to be unnecessary; in $0.2 \mathrm{M}$ aqueous methylamine at $\mathrm{pH} 8 \cdot 6$, retinylidenemethylamine does not hydrolyse to retinene.

This will account for the stability of alkaline indicator yellow produced from rhodopsin solution as there will be a large number of amino groups in opsin and other retinal proteins which will prevent hydrolysis of the Schiff's base. Confirmation of this can be found in the report by Collins (1953), that when all free amino groups in rhodopsin preparations were 'blocked' by formaldehyde, alkaline indicator yellow solutions hydrolysed very rapidly. There is therefore no essential difference in the stability of retinylidenemethylamine and of indicator yellow obtained from the eye.

Ball et al. (1949) found that methylamine in acid solution will not react with retinene to give retinylidenemethylamine. The most likely explanation of this is that the methylammonium ion is ineffective in equilibrium (1). Methylamine is half ionized at about $\mathrm{pH} \mathrm{10.5} \mathrm{(Clark,} \mathrm{1928),} \mathrm{and,} \mathrm{if} \mathrm{this} \mathrm{explanation}$ is true, the stabilizing action of a large excess of methylamine on the Schiff's base in alkaline solution should become increasingly less effective as the $\mathrm{pH}$ falls. This was tested by making an aqueous solution of retinylidenemethylamine in $0.2 \mathrm{M}$ methylamine and adjusting the $\mathrm{pH}$ to values of $6 \cdot 3$ and $3 \cdot 7$. Results are shown in Table 2. Comparing the rates of hydrolysis in the presence and absence of excess methylamine, it is seen that at $\mathrm{pH} 8 \cdot 6$, excess methylamine prevents hydrolysis; at $\mathrm{pH} 6 \cdot 3$ it slows hydrolysis, which nevertheless proceeds quite rapidly; at $\mathrm{pH} 3 \cdot 7$, excess methylamine has 
no effect. These findings agree with the suggestion that only un-ionized methylamine is effective in equilibrium (1).

Further, they will explain why, in the absence of added methylamine, the hydrolysis of retinylidenemethylamine slows as the $\mathrm{pH}$ rises from $6 \cdot 6$ to $8 \cdot 6$, whereas changing the $\mathrm{pH}$ from $8 \cdot 6$ to 13 has a much smaller effect (see Table 2). On putting retinylidenemethylamine into water at $\mathrm{pH} \mathrm{13,} \mathrm{it} \mathrm{breaks}$ down to retinene and methylamine, but the rate of the reaction will be retarded as free methylamine accumulates. In less alkaline solutions, the methylamine formed by the hydrolysis will be converted partly (the extent depending on $\mathrm{pH}$ ) into methylammonium ions, which will not be effective in slowing the overall rate of breakdown.

If this is true, then any conditions which remove free methylamine from the system will similarly quicken hydrolysis. The breakdown of retinylidenemethylamine was investigated in an aqueous solution of $\mathrm{pH} 8.6$ containing $4 \%(\mathrm{w} / \mathrm{v})$ formaldehyde, as a trapping agent for amino groups. This produced a very rapid hydrolysis - faster than that recorded at any $\mathrm{pH}$ value (see Table 2).

These results all agree with the explanation proposed for the variation in the rate of hydrolysis of retinylidenemethylamine in solutions of different alkalinity. They also clarify the report of Collins (1953) that alkaline indicator yellow hydrolysed much faster than did retinylidenemethylamine. His alkaline indicator yellow was produced in the presence of $30 \%(\mathrm{w} / \mathrm{v})$ formaldehyde and would therefore appear to break down faster than retinylidenemethylamine, which he studied in a solution containing no formaldehyde.

Although retinylidenemethylammonium salts do not hydrolyse at $\mathrm{pH} 1$, they break down to retinene more easily as the acidity falls. This can be explained by assuming that whereas the uncharged Schiff's base retinylidenemethylamine is readily hydrolysed, the retinylidenemethylammonium ion is stable. At pH 1 substantially all the retinylidenemethylamine will be converted into retinylidenemethylammonium ions, i.e. equilibrium (2) will be forced to the left by the high concentration of hydrogen ions.

$$
\mathrm{C}_{19} \mathrm{H}_{27} \mathrm{CH}=\mathrm{N}^{+} \mathrm{H} \cdot \mathrm{R} \rightleftharpoons \mathrm{C}_{19} \mathrm{H}_{27} \mathrm{CH}=\mathrm{N} \cdot \mathrm{R}+\mathrm{H}^{+} \text {. }
$$

As the $\mathrm{pH}$ of the solution rises, there will be more retinylidenemethylamine itself present, which will hydrolyse to retinene. More retinylidenemethylammonium ions will change over to retinylidenemethylamine and so give retinene until all the retinylidenemethylammonium ions have disappeared. The limiting factor will be the concentration of un-ionized retinylidenemethylamine. The higher the $\mathrm{pH}$, the more retinylidenemethylamine will be present and the faster the apparent hydrolysis of the retinylidenemethylammonium salt to retinene.

The concentrations of the uncharged Schiff's base must be low, as absorption curves recorded during the hydrolysis of retinylidenemethylammonium acetate at $\mathrm{pH} 3.7$ (Fig. 2) show an isosbestic point indicating that only two absorbing substances are present in detectable quantities, i.e. retinylidenemethylammonium acetate and retinene.

These data on retinylidenemethylamine may be used to explain the stability of indicator yellow in solutions prepared from eyes. In alkaline solution the excess of free amino groups in opsin and any other proteins present maintains equilibrium (1) in favour of alkaline indicator yellow, i.e. alkaline indicator yellow is stable. In fairly strongly acid solutions, the high hydrogen-ion concentration maintains equilibrium (2) in favour of the stable acid indicator yellow. In less acid conditions, any uncharged Schiff's base molecules present will hydrolyse to retinene, as in the $\mathrm{pH}$ range over which acid indicator yellow will exist, the amino groups necessary for stabilizing any alkaline indicator yellow molecules will have been ionized and thus made ineffective. Therefore in less acid conditions, acid indicator yellow will appear unstable. This theory enables all observations of previous workers on the stability of indicator yellow to be explained in simple chemical terms. There is no need for the postulate (Lythgoe, 1937) of two forms of acid indicator yellow. There are slight differences in the stability of the analogue and of the naturally occurring material, e.g. indicator yellow is stable at pH 3.3 (Lythgoe, 1937), while retinylidenemethylamine will hydrolyse at this $\mathrm{pH}$. Such differences may be expected; the range of stability of these substances will depend on the $\mathrm{p} K_{a}$ of the Schiff's base; which is likely to vary with the amine concerned, and also on the $\mathrm{p} K_{a}$ values of the amino groups in the rhodopsin solutions.

\section{Acid indicator yellow and rhodopsin}

Lythgoe (1937) found that if rhodopsin solutions are brought to $\mathrm{pH} \mathrm{4.0}$ in the dark, indicator yellow is formed; that is, acid and light appear to have the same effect on rhodopsin in the conditions used. Collins \& Morton (1950) have shown that, when formed in this way, acid indicator yellow must be a direct derivative of rhodopsin. Lythgoe (1937) found the extinction at $440 \mathrm{~m} \mu$. after acid destruction of frog rhodopsin to be 0.90 of that initially observed at $502 \mathrm{~m} \mu$. in rhodopsin. Wald \& Brown (1953) have found $\epsilon(500 \mathrm{~m} \mu$.) of ox rhodopsin to be 40600 , and Pitt et al. (1950) have reported $\epsilon$ $(440 \mathrm{~m} \mu$.) of retinylidenemethylammonium salts as 41000 . The percentage of the retinene present in 
rhodopsin which was recovered in indicator yellow can therefore be estimated from the relationship:

$$
\text { Percentage recovery }=\frac{40600}{41000} \times \frac{E_{\mathrm{Y}}}{E_{\mathrm{B}}} \times 100,
$$

where $E_{\mathbf{R}}=$ extinction at $500 \mathrm{~m} \mu$. of rhodopsin solution, $E_{\mathrm{y}}=$ extinction at $440 \mathrm{~m} \mu$. after acid destruction of rhodopsin (after correcting for dilution).

By using this, it can be calculated from the data of Lythgoe (1937) that by adding an acid buffer of pH 4, $89 \%$ of the retinene present in rhodopsin was converted into acid indicator yellow. However, this figure rests on the following assumptions: (1) $\epsilon_{\max }$, of ox rhodopsin is the same as that of frog rhodopsin. (2) $\epsilon_{\max }$ of retinylidenemethylammonium chloride is the same as that of acid indicator yellow. (3) The retinylidene groups of acid indicator yellow have the same proportion of cis and trans forms as in the solutions of retinylidenemethylammonium chloride used by Pitt et al. (1955) to determine the $\epsilon_{\max }$. value used in the above relationship. (4) No alkaline indicator yellow was present in Lythgoe's rhodopsin solutions.

Assumption (2) is probably justified. $\epsilon_{\max }$ values for the indicator yellow analogue agree quite well with those of acid indicator yellow formed by the reaction of retinene with retinal-protein solutions (Collins \& Morton, 1950). There is no reason to believe that assumption (1) will introduce any large error, but it is desirable to repeat Lythgoe's work using solutions of ox rhodopsin, in particular by acidifying to a pH below 4, as, in Lythgoe's work, the acid indicator yellow was, of course, unstable. Further, it is essential to exclude the possibility of error arising from the presence of any alkaline indicator yellow in the rhodopsin solution. On acidification, this would change to acid indicator yellow, not derived directly from rhodopsin, and so give misleadingly high figures. Lythgoe was unable to check this point as his spectrophotometer did not read below $395 \mathrm{~m} \mu$.

To avoid the presence of alkaline indicator yellow in the rhodopsin solutions prepared for this experiment, rods from ox eyes, after alum treatment, were washed with a buffer of pH $6 \cdot 3$ (converting any indicator yellow into free retinene), dried with anhydrous sodium sulphate and extracted with light petroleum to remove this retinene. Finally, the rhodopsin was extracted with digitonin in a buffer solution of $\mathrm{pH} \mathrm{6.3,} \mathrm{as} \mathrm{retinylideneamines} \mathrm{cannot} \mathrm{form} \mathrm{at} \mathrm{this} \mathrm{pH}$.

To the solution of ox rhodopsin buffered at $\mathrm{pH} 6 \cdot 3$, acid was added bringing the $\mathrm{pH}$ to below $1 ; \lambda_{\max }$ moved to $440 \mathrm{~m} \mu$., showing formation of indicator yellow. This must have come entirely from rhodopsin, since any retinene which might have been present other than in the rhodopsin chromophore would not have been able to form indicator yellow at $\mathrm{pH} 6 \cdot 3$. Of the retinene present in rhodopsin, $92 \%$ was recovered in acid indicator yellow.
To confirm this, it was desirable to check that no appreciable amount of free retinene was formed by the acid destruction of rhodopsin. This was first attempted by bringing the $\mathrm{pH}$ to about 6 (when acid indicator yellow hydrolyses to retinene), and estimating the total amount of free retinene present. The method was not very reliable; digitonin began to precipitate in acid solution, causing turbidity with consequent inaccuracies in measurements of extinction. To minimize this source of error, it was found advisable to determine free retinene by using a wavelength on the long. wave side of $400 \mathrm{~m} \mu$. The most suitable method was to measure it as a retinylidenemethylammonium salt.

After acid destruction of rhodopsin, the extinction at $440 \mathrm{~m} \mu$. was measured, the mixture made alkaline with $\mathrm{KOH}$ containing a great excess of methylamine, to convert both the acid indicator yellow and any free retinene into retinylidenemethylamine. The solution was reacidified and the extinction at $440 \mathrm{~m} \mu$. again read to determine the amount of retinylidenemethylammonium salt now present. Had any free retinene been formed in the acid destruction of rhodopsin, the second reading of the extinction at $440 \mathrm{~m} \mu$. would have been higher (after correcting for dilution) than the first. No such increase was found; the final amount of retinylidenemethylammonium chloride present was $98 \%$ of the indicator yellow formed after acid destruction of retinene, a difference which is probably within experimental error.

When rhodopsin is converted by acid into acid indicator yellow, no free retinene, therefore, is formed; all the retinene of the rhodopsin chromophore must be present in acid indicator yellow. The recovery of retinene in indicator yellow would therefore be expected to be $100 \%$. Part of the difference between this and the experimentally found figure of $92 \%$ probably arises from the incorrectness of assumption (3) made above. Pitt et al. (1955) determined $\epsilon_{\max }$ of retinylidenemethylammonium chloride by dissolving the crystalline all-trans form in dilute acid. There is no certainty as to the configuration of the retinylidene group in acid indicator yellow when formed by acid destruction of rhodopsin. It may well contain a proportion of cis isomers, as rhodopsin is formed from a cis-retinene (Hubbard \& Wald, 1952), which will have a lower $\epsilon_{\max }$ than the all-trans form. If this is so, then a $100 \%$ recovery (in terms of the above relationship) is impossible. It seems likely that this is the cause of the apparently low recovery; when repeated on a different rhodopsin preparation, the experiment gave the same result.

\section{DISCUSSION}

\section{Indicator yellow and rhodopsin}

Two opinions have been held of the status of indicator yellow: $(a)$ that it is a true derivative of rhodopsin, and (b) that it is formed by a casual combination of retinene with amino groups. Both views have found supporters. The question has been discussed by Collins (1953), who has shown that 
indicator yellow may be a true derivative of rhodopsin. The point is important: the hypothesis that retinene is joined to opsin by a carbon-nitrogen link (Collins \& Morton, 1950; Collins, 1953) rests on the assumption that indicator yellow can be derived directly from rhodopsin.

The present study of the stability of retinylidenemethylamine-and by analogy of indicator yellowshould be considered side by side with the findings of Collins \& Morton (1950) on the interaction of retinene and retinal proteins. It is perhaps opportune to reassess the nature and status of indicator yellow obtained from rhodopsin solutions.

In alkaline solution, indicator yellow is stabilized by the excess of free amino groups in opsin and in other retinal proteins present as impurities in most rhodopsin preparations. Any retinene formed by hydrolysis of alkaline indicator yellow will immediately reform a Schiff's base by uniting with one of these amino groups, which may well not be the one to which it was joined in rhodopsin.

However, since the ionized amino groups of retinal proteins formed in acid are ineffective in uniting with retinene, any indicator yellow produced from rhodopsin in acid solution must have its retinylidene group still attached to the nitrogen atom involved in the retinene-opsin linkage (Collins \& Morton, 1950).

Indicator yellow is, then, a true derivative of rhodopsin provided that it has not at any time passed through alkaline solution containing an excess of amino groups. If it has, it is probably a mixture of various retinylideneamines. This view should harmonize the differing opinions of indicator yellow expressed in the past.

Most of the published work on indicator yellow has been carried out on solutions of rhodopsin. Recently, Arden (1954) has studied the photochemical destruction (bleaching) of rhodopsin in intact rods from frog eyes and has found no evidence of the existence of indicator yellow as an intermediate in the production of retinene. Yet abundant proof exists that indicator yellow can be formed from rhodopsin solutions in suitable conditions. The conclusion to be drawn from Arden's work must be that the $\mathrm{pH}$ of the frog rod is within the range in which indicator yellow is not stable. Arden studied the results of bleaching rhodopsin in rods suspended in solutions of various $\mathrm{pH}$ values, but still found no difference in the course of bleach. ing. Presumably putting rods in buffers had no appreciable effect on the $\mathrm{pH}$ of the interior of the rod-a likely assumption considering the relative impermeability of the frog rod sheath (Arden, 1954).

Arden's results show that in the visual cycle in the rods indicator yellow is functionally negligible, but that does not detract from the finding that indicator yellow may be a true derivative of rhodopsin.

Collins (1953) showed this to be so by exposing rhodopsin to light in the presence of formaldehyde to prevent recombination of retinene with other amino groups. He was able to detect less than half the indicator yellow expected; the low recovery was due largely to the rapid hydrolysis of indicator yellow in these conditions.

Our repetition of the work of Lythgoe (1937) in the light of more recent knowledge has shown that rhodopsin can be converted directly and quantitatively into acid indicator yellow ; $N$-retinylideneopsin has been formed with the retinylidene group still attached to the nitrogen atom involved in the retinene-opsin link. Provided that the $\mathrm{pH}$ is kept down to 1 ; the retinylidene-opsin ammonium salt formed is fairly stable. This is the first unequivocal demonstration of a direct derivative of rhodopsin stable in aqueous solution at room temperature, and which still contains the opsin portion of the molecule. It is hoped to exploit this to identify the amino group to which retinene is linked in rhodopsin.

\section{Nomenclature}

Wald (1953) has objected to the use of the name 'indicator yellow' and urged its replacement by a more 'chemical' term. A revised nomenclature for retinene derivatives was proposed by Pitt et al. (1955) and has been used throughout this paper. We suggest that the general name 'indicator yellow' be retained for the mixture of Schiff's bases formed from bleached rhodopsin which has passed through alkaline solution. When indicator yellow is obtained in conditions in which no migration of retinylidene groups can occur, this true derivative of rhodopsin is better named $N$-retinylideneopsin.

\section{The carbon to nitrogen link in rhodopsin}

Acid destruction of rhodopsin converts its retinene residue into a substituted ammonium salt form of retinylideneopsin. Every retinylidene group in the salt is joined to a nitrogen atom, and so every retinene residue in rhodopsin must have been linked to opsin by a carbon-nitrogen bond. This bond must readily give rise to the azomethine link of retinylideneopsin. It is difficult to conceive how the $-\mathrm{C}=\mathrm{N}-$ grouping could be formed from any other carbon-nitrogen linkage in the conditions in which $N$-retinylideneopsin is produced from rhodopsin. The simplest possibility is that it already exists in rhodopsin.

$N$-Retinylideneopsin has indicator properties because the nitrogen of the azomethine group can alternate between the tertiary and quaternary states. The spectrum of rhodopsin does not change with pH (Lythgoe, 1937), and Lythgoe \& Quilliam (1938) have shown that transient orange-in modern 
terms, probably a mixture of lumi- and metarhodopsin (Wald, Durell \& St George, 1950)—does not show indicator behaviour. If, in all these compounds, the terminal carbon atom of retinene is linked to a nitrogen atom of opsin by a double bond, then to account for their lack of indicator properties, the nitrogen atom must be either fixed in the quaternary state or not involved directly in the effective chromophore of rhodopsin.

\section{SUMMARY}

1. The stability of retinylidenemethylamine, an indicator yellow analogue, has been studied in aqueous solutions over a range of $\mathrm{pH}$ values.

2. It is concluded that retinylidenemethylammonium ions are stable, but that uncharged retinylidenemethylamine molecules hydrolyse to retinene. This hydrolysis is prevented by the presence of excess methylamine, but not by methylammonium ions.

3. These findings explain the influence of $\mathrm{pH}$ on the stability of indicator yellow solutions obtained from eyes.

4. On adding acid to rhodopsin solutions in the dark, stable acid indicator yellow solutions can be formed, with the retinene residue attached to the same nitrogen atom as it is in rhodopsin.

5. Essentially all the retinene residues in the rhodopsin chromophore have been shown to be attached to a nitrogen atom of opsin.

6. The status of the different types of indicator yellow formed from rhodopsin solutions is assessed.

We are indebted to the Medical Research Council for financial assistance.

\section{REFERENCES}

Arden, G. B. (1954). J. Physiol. 123, 386.

Ball, S., Collins, F. D., Dalvi, P. D. \& Morton, R. A. (1949). Biochem. J. 45, 304.

Bliss, A. F. (1948). J. biol. Chem. 172, 165.

Bliss, A. F. (1951). Arch. Biochem. Biophys. 31, 197.

Clark, W. M. (1928). The Determination of Hydrogen Ions, 3rd ed. London: Baillière, Tindall and Cox.

Collins, F. D. (1953). Nature, Lond., 171, 469.

Collins, F. D., Love, R. M. \& Morton, R. A. (1952). Biochem. J. 51, 292.

Collins, F. D. \& Morton, R. A. (1950). Biochem. J. 47, 10. Hubbard, R. \& Wald, G. (1952). J. gen. Physiol. 36, 269.

Lythgoe, R. J. (1937). J. Physiol. 89, 331.

Lythgoe, R. J. \& Quilliam, J. P. (1938). J. Physiol. 93, 24. Pitt, G. A. J., Collins, F. D., Morton, R. A. \& Stok, P. (1955). Biochem. J. 59, 122.

Wald, G. (1938). J. gen. Physiol. 21, 795.

Wald, G. (1953). Annu. Rev. Biochem. 22, 497.

Wald, G. \& Brown, P. K. (1953). J. gen. Physiol. 37, 189.

Wald, G., Durell, J. \& St George, R. C. C. (1950). Science, 111, 179.

Zechmeister, L. (1944). Chem. Rev. 34, 267.

\title{
A Modified Conway Unit for Microdiffusion Analysis
}

\author{
BY K. J. ÖBRINK \\ The Institute of Physiology, University of Uppsala, Sweden
}

(Received 21 June 1954)

\begin{abstract}
Absorption by the fixative is a difficulty in the microdiffusion method (Conway, 1950) for the determination of small amounts of bromide. Conway recommends a mixture of solid and liquid paraffin as fixative, but points out that the quality of the paraffin is important and that the glass lids should be only lightly smeared. However, it has been found that a definite amount of bromine (sometimes as much as 0.10 m-mole) was always absorbed by the paraffins available here. A similar difficulty with chloride determinations induced Gordon (1952) to use a chlorinated fixative. Pirt \& Chain (1952) avoided such small losses of halogen by using a silicone fixative.

A slight modification of the unit is described which avoids any such difficulty and is also more suitable for analytical investigations where relatively high temperatures are required.
\end{abstract}

\section{EXPERIMENTAL AND RESULTS}

The modified unit has an extra chamber (the closing chamber) which is half-filled with the same solutions as the outer diffusion chamber, with the exception of the fluid to be analysed. (In the case of bromide determinations a saturated potassium dichromate solution in $20 \%(\mathrm{v} / \mathrm{v})$ $\mathrm{H}_{2} \mathrm{SO}_{4}$ was used.) A liquid trap is formed by dipping an inverted Petri dish into this chamber and thus closing the Unit immediately after running-in the liberating substance. A similar principle of a liquid trap for closing the Unit was developed by Kinsey \& Robison (1946), who used oil in the trap. Their Unit was, however, quite different from Conway's and consisted of only one diffusion chamber.

Figs. 1 and 2 show the modified Unit with the cover. In the Unit illustrated in Fig. 2 the closing chamber was made from acrylic plastic (Plexiglass) and attached to the Units by a plastic fixative. If Conway Units are not available for modification, Units containing all three chambers can easily be constructed of one material and no surface requires 